\title{
XII. On a method of determining the sensibility of a balance
}

\author{
J.H. Poynting Sc.D. F.R.S. \& G.W. Todd M.Sc.
}

To cite this article: J.H. Poynting Sc.D. F.R.S. \& G.W. Todd M.Sc. (1909) XII. On a method of determining the sensibility of a balance , Philosophical Magazine Series 6, 18:103, 132-135, DOI: $10.1080 / 14786440708636677$

To link to this article: http://dx.doi.org/10.1080/14786440708636677

曲 Published online: 21 Apr 2009.

Submit your article to this journal $₫$

Џ Article views: 3

Q View related articles $\square$

Citing articles: 1 View citing articles $\square$ 
become more penetrating than the radiation characteristic of the absorber, this increase in the absorption being intimately connected with the emission of tertiary radiation by the absorber in these circumstances (see paper on "The Absorption of X-Rays," Barlsla \& Sadler, Phil. Mag. May 1909).

The fraction of this increase in the absorption of the energy of the secondary beam, which is re-emitted as tertiary radiation, is not constant, but decreases as the secondary beam becomes more penetrating, slowly at first, and then more rapidly when a very penetrating secondary beam is used.

In conclusion I wish to thank Dr. Barkla for the interest he has shown throughout this research, and especially for his kindly criticism and advice during the writing of this paper.

George Holt Physics Laboratory,

University of Liverpool, 24th March, 1909.

XII. On a Method of Determining the Sensibility of a Balance. $B y$ J. H. Poynting, Sc.II., F.R.S., and G. W. Todd, M.Sc.*

T $N$ the method, as we have arranged it, a small frame 1 (fig. 1, end view) is fixed at the centre of the beam of a 16-inch Oertling balance. This carries two $V_{s}$ about $2 \mathrm{~cm}$.

Fig. 1.

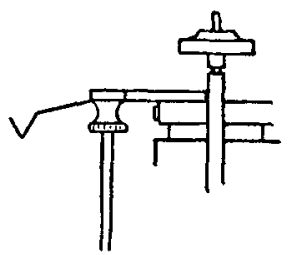

End view of $V$ frame fixed to balance-beam.

apart, and in the $V_{s}$ lies a straight wire or fibre about $3 \frac{1}{2} \mathrm{~cm}$. long, parallel to the beam and level with the central knifeedge. This wire takes the place of the ordinary rider, and

* Communicated by the Physical Society : read June 25, 1909. 
we shall call it "the rider." Its weight is determined before use as accurately as possible by weighing on an assay balance. The sensibility is determined by moving the rider either to right or left a mensured distance. If this distance is $d$, if the half length of beam is $b$, and if the weight of the rider is $R$, the movement is equivalent to an addition of weight to one pan, $\mathrm{R} d / b$.

In order to move the rider a definite distance a stout horizontal rod (fig. 2) passes through the balance-case from side to side without contact with the case, and is supported at its ends outside, and independent of, the case. It is parallel to the beam and a little lower than the $V$ frame. On the rod are fixed horizontally two Brown \& Sharp micrometer-screws divided to $.01 \mathrm{~mm}$. and allowing an estimate of $.001 \mathrm{~mm}$.

Fig. 2.

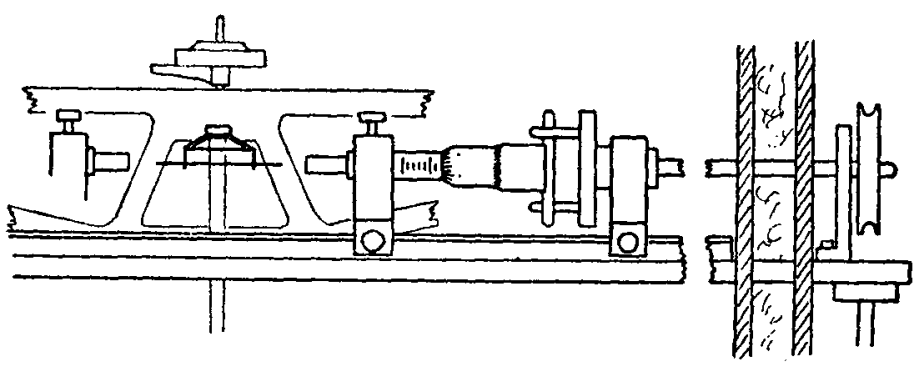

Arrangement of right-hand micrometer-screw.

Their axes are in one line coinciding with the axis of the rider, and they are fixed so that one can bear against one end and the other against the other end of the rider. Their ends are plane and the ends of the rider are bluntly pointed. Each micrometer screw-head has a cross piece fixed on it, and a fork which can be rotated about an axis in the continuation of the axis of the screw by a pulley outside the case, can engage with the cross piece, and so advance or withdraw the screw. The pulley is worked by an endless string passing to a pulley at the side of the observer, who is about 2 metres in tront of the bulance. The micrometer divisions are illuminated and each micrometer is viewed by its own telescope. The position of the balance-beam is read by a double-suspension mirror, telescope and scile. The scale is divided to millimetres and is about 3 metres from the 
mirror. The double-suspension mirror is fully described in the Phil. Trans. A, 1891, p. 572. It is of course not essential to the method, but was chosen because of the great magnification of the deflexion which it gives.

Let us suppose that the value of the scale-divisions of the deflexion is to be determined by a movement of the rider from right to left. The two micrometer-screws are withdrawn so that neither is in contact with the rider, that on the left so far that the rider will not touch it in its subsequent travel. The beam is lowered and allowed to swing. Then the right-hand screw is advanced till it bears against the end of the rider and pushes it some small distance. The contact is seen to have occurred by the interference with freedom of swing, as watched in the telescope. The micrometer is then read. Let its reading be $m_{1}$. It is then withdrawn a little so as to leave the rider free, and the centre of swing $C_{1}$ is determined in the usual way from three successive turning points. Then the micrometer is advanced again so as to push the rod a little further, and its reading $m_{2}$ is taken. It is then withdrawn and the new centre of swing $\mathrm{C}_{2}$ is taken. If $m_{1}-m_{2}=d, \mathrm{C}_{1}-\mathrm{C}_{2}$ divisions deflexion are due to an addition of $\mathrm{R} d / b$ to the lelt pan.

The right-hand micrometer may then be withdrawn and the left-hand micrometer may be brought into action in a similar manner, and so on, the two screws being used alternately.

The balance-case was fixed on a shelf and was enclosed in a tin-foiled wood box with wool loosely packed between box and case. The case and box were provided with plateglass windows to view the mirror and the micrometer divisions. The following abstract of some determinations of sensibility will serve to show what accuracy may be attained :-

I. Rider German silver wire, 7.35 mgm. Half length of beam, $20 \cdot 272 \mathrm{~cm}$. 10 determinations alternately left and right. Mean travel of rider ........... 2.4850 mm Mean deflexion ............... 21.26 divisions. Mean value for 20 divisions ... $0.0848 \mathrm{mgm}$. The separate determinations range between 20 divisions $=0.0877$

$$
\text { and } 20 \quad "=0.0824 \text {. }
$$


II. The same rider.

10 determinations alternately left and right.

Mean travel of rider .......... $5 \cdot 2713 \mathrm{~mm}$.

Mean deflexion ............... $45 \cdot 47$ divisions.

Mean value for 40 divisions ... $0 \cdot 1681 \mathrm{mgm}$.

The separate determinations range between

40 divisions $=0 \cdot 1722$

$$
\text { and } 40 \quad, \quad=0.1632 \text {. }
$$

III. Rider German silver wire, 189.05 mgm.

7 determinations alternately left and right.

Mean travel of rider ........... $0 \cdot 1764 \mathrm{~mm}$.

Mean deflexion ................ 38.70 divisions.

Mean value for 40 divisions ... $0 \cdot 1691 \mathrm{mgm}$.

The separate determinations range between

40 divisions $=0 \cdot 1709$

and $40, \quad=0.1654$.

IV. The same rider.

7 determinations alternately left and right.

Mean travel of rider ............ 0.3004 mm.

Mean deflexion ............... 64.84 divisions.

Mean value for 60 divisions ... $0.2578 \mathrm{mgm}$.

The separate determinations range between

60 divisions $=0 \cdot 2612$

and $60 \quad, \quad=0 \cdot 2518$.

XIII. The Balance as a Sensitive Barometer. By G. W. Topd, M.Sc.*

TT occurred to the author while testing with Professor Poynting the accuracy of a new method of determining the sensibility of a balance, described on p. 132, in which a thin rod or fibre is used instead of the usual rider, that a balance might with proper precautions be converted into a very delicate barometer.

A difference in volume on the opposite sides of a balance will give rise to motions of the pointer when the density of the surrounding air changes. Small oscillations of the pointer, due chiefly to convection currents, become negligible if this difference in volume is sufficiently large. Alterations in the density of the air are produced by changes in pressure, in temperature, and in the percentage of aqueous vapour in

- Communicated by the Physical Society : read June 25, 1909. 